\title{
Current oscillations in a metallic ring threaded by a time-dependent magnetic flux.
}

\author{
Liliana Arrachea \\ Scuola Internazionale Superiore di Studi Avanzati \\ Via Beirut n. 2-4 (34013) Trieste, Italy. \\ and \\ Departamento de Física FCEyN, Universidad de Buenos Aires \\ Pabellón I, Ciudad Universitaria (1428) Buenos Aires, Argentina.
}

\begin{abstract}
We study a mesoscopic metallic ring threaded by a magnetic flux which varies linearly in time $\Phi_{M}(t)=\Phi t$ with a formalism based in Baym-Kadanoff-Keldysh non-equilibrium Green functions. We propose a method to calculate the Green functions in real space and we consider an experimental setup to investigate the dynamics of the ring by recourse to a transport experiment. This consists in a single lead connecting the ring to a particle reservoir. We show that different dynamical regimes are attained depending on the ratio $\hbar \Phi / \Phi_{0} W$, being $\Phi_{0}=h c / e$ and $W$, the bandwidth of the ring. For moderate lengths of the ring, a stationary regime is achieved for $\hbar \Phi / \Phi_{0}>W$. In the opposite case with $\hbar \Phi / \Phi_{0} \leq W$, the effect of Bloch oscillations driven by the induced electric field manifests itself in the transport properties of the system. In particular, we show that in this time-dependent regime a tunneling current oscillating in time with a period $\tau=2 \pi \Phi_{0} / \Phi$ can be measured in the lead. We also analyze the resistive effect introduced by inelastic scattering due to the coupling to the external reservoir.

Pacs Numbers:73.40.Gk, 73.20.Dx,72.10.-d
\end{abstract}

\section{INTRODUCTION}

In the last years, the progress of technology has enabled the experimental study of very interesting phenomena taking place in mesoscopic systems. The fascinating feature in these systems is that several physical processes that have a pure quantum-mechanical origin can be observed in a suitable transport experiment. Paradigmatic examples are the Aharanov-Bohm effect in systems with annular geometry and Bloch oscillations in periodic structures.

The Aharanov-Bohm effect in circular devices motivated a vast theoretical and experimental activity for some time now. A metallic mesoscopic ring threaded by a static magnetic field displays persistent currents, which oscillate as a function of the magnetic flux $\Phi_{M}$, with a period $\Phi_{0}=2 \pi h / e$ [1]. Like other mesoscopic phenomena, these persistent currents are a consequence of the coherence of the wave function throughout the system. The Aharanov-Bohm effect also manifests itself when such a ring is connected to two metallic leads at different external voltages. This geometry is usually employed in experiments to measure the conductance of the metallic ring in the presence of the external flux. In this case, quantum interference cause oscillations in the current through the collector lead with a period $\Phi_{0}$ as $\Phi_{M}$ varies [2].

More than 70 years ago, Bloch predicted the occurrence of a time-periodic motion of the electrons in a band under the influence of an external constant electric field $F$ [3]. Recently, Bloch oscillations with a period $h / e F L$ has been observed in superlattices [A], being $L$ the periodicity of the lattice and $e$ the electron charge. This motivated new theoretical development in the field 5 [7].

A metallic ring penetrated by a time-dependent magnetic field is a very interesting system, worth of theoretical and experimental study. Since time is the unavoidable variable in any experimental measurement of currents while the magnetic field is varied, that device is more realistic than its static-field counterpart. The case of a magnetic flux with a linear time dependence is particularly appealing because this situation is closely related with that of electrons in a periodic lattice under the influence of a dc voltage. In fact, a magnetic flux that varies linearly in time induces a constant electric field $F=(1 / c L) d \Phi_{M}(t) / d t$, being $L$ the longitude of the ring, while the geometry of the ring produces a periodic structure for the electrons inside it. While a good amount of literature can be found on the Aharanov-Bohm effect, considerably fewer studies have been reported on this problem. It has been addressed in an early work by Büttiker, Imry and Landauer [8] who predicted that the electrons in an ideal one-dimensional ring should execute Bloch oscillations driven by the induced electric field. Some time later, Landauer and Büttiker showed that inelastic scattering in that system generates a dc current and dissipation [9]. A single lead connected between the ring and an external reservoir was later proposed by Büttiker as a concrete way to introduce inelastic scattering events [10]. Since then, the attention focused mainly in Aharanov-Bohm loops where the flux threading the ring is static and devices with inserted quantum dots seem to occupy the center of the stage [11]. 
In this work the problem of the normal-metal loop in the presence of an external time dependent magnetic flux with the form $\Phi_{M}(t)=\Phi t$ is studied in the framework of non-equilibrium Green functions. We consider an ideal ring described by a one-dimensional tight-binding model with a bandwidth $W$ and we propose an approach to calculate the Green functions in real space. This procedure enables to gain insight on the different energy scales that intervene in the dynamics of the problem. In addition, it has the advantage of being also adequate to tackle more general situations where the configuration is not translational invariant, like the case of rings with disorder and with inserted barriers or quantum dots.

The experimental study of the physical properties of the ring in the presence of the variable magnetic field requires some kind of coupling between this system and the external world. We consider a concrete experimental setup to study time-dependent charge oscillations in the ring. This consists in a single lead connecting it to a particle reservoir. The goal is to study the quantum tunneling between the ring and the reservoir, originated by the time dependent oscillations driven by the induced electric field. The metallic wire is described as an ideal system of non-interacting electrons and the reservoir is assumed to fix the chemical potential $\mu$. We use an approach based in Baym-KadanoffKeldysh formalism to solve the coupled system. This method has been successful to derive generalized transport equations [12,13] in systems with an explicit time dependence. In the present context the main idea is to represent the effect of the lead and the reservoir by the self-energy effects they produce.

In the case of pure one-dimensional rings pierced by a static magnetic field in the absence of disorder, the persistent currents vanish as $1 / L$, being $L$ the circumference of the ring [14]. In the time dependent case there is a non trivial connection between the size of the system and its dynamical behavior. We study the behavior of the retarded Green function as a function of $\Phi$ and $L$. We argue that for a magnetic field varying at a rate such that $h \Phi / \Phi_{0} \leq W$, the time dependent features of the relevant physical magnitudes vanish as $1 / L$. Instead, for more rapid changes of the magnetic field such that $h \Phi / \Phi_{0}>W$, their time dependence vanish exponentially fast as a function of $L$. Our aim is to show that for moderate lengths $L$ and field variations satisfying the condition $h \Phi / \Phi_{0} \leq W$, quantum tunneling takes place between the ring and the reservoir and a time dependent current could be measured in the lead. We also analyze the effect that inelastic scattering originated by the coupling to the reservoir cause on the transport properties of this system.

The paper is organized as follows. Section II is devoted to expose the theoretical approach. In Section III we obtain the set of equations to calculate the retarded Green function in the ring unconnected from the reservoir as well as connected to it. Results are shown in Section IV. We finally summarize our study and present the conclusions in Section V.

\section{BASIC THEORY}

We consider an experimental setup to study the dynamics of the ring in the presence of the time-dependent magnetic flux of the form $\Phi_{M}=\Phi t$. The device is sketched in Fig. 1a. Our aim is to study the quantum tunneling between the ring and an external particle reservoir by measuring the current flowing through the lead. We use an approach based in Baym-Kadanoff-Keldysh non-equilibrium Green functions to solve the coupled system. We follow a procedure similar to that used in Refs. [12,13], where the effect of the lead and the reservoir is represented by a suitable self-energy. The system is simplified as indicated in Fig. 1b. The lead and the reservoir are described as an effective system $\alpha$ with a fixed chemical potential $\mu$. A contact, described by a single bond, establishes the connection between the ring and the lead.

The total hamiltonian of this system contains three pieces:

$$
H=H_{\text {ring }}+H_{\alpha}+H_{\text {contact }}
$$

The first term describes the metal ring threaded by the magnetic flux. We consider non-interacting spinless electrons in a tight-binding chain of longitude $L$ with $N$ sites, lattice parameter $a=L / N$ and periodic boundary conditions $(N+1 \equiv 1)$. The model hamiltonian is

$$
H_{\text {ring }}=-T \sum_{l}^{N}\left(e^{-i \phi t} c_{l}^{\dagger} c_{l+1}+e^{i \phi t} c_{l+1}^{\dagger} c_{l}\right),
$$

with $l=1, \ldots, N$ and $\phi=\Phi /\left(\Phi_{0} N\right)$, being $T$ the hopping matrix element between nearest neighbors. The timedependent phase $\phi t$ attached to each link accounts for the presence of the external magnetic flux. The term $H_{\alpha}$ represents the lead and the reservoir. For the moment we do not give any explicit hamiltonian for this system. 
It is enough to assume that it is an infinite system of non-interacting electrons with a chemical potential $\mu$. The hamiltonian describing the contact is

$$
H_{\text {contact }}=-T_{1 \alpha}\left(c_{1}^{\dagger} c_{\alpha}+c_{\alpha}^{\dagger} c_{1}\right)
$$

In terms of Green functions, the current through the contact is written as

$$
J_{1 \alpha}=\frac{2 e}{\hbar} R e\left[T_{1 \alpha} G_{1 \alpha}^{<}(t, t)\right]
$$

where $G_{1 \alpha}^{<}\left(t, t^{\prime}\right)=i\left\langle c_{1}^{\dagger}(t) c_{\alpha}\left(t^{\prime}\right)\right\rangle$. The latter obeys the following equation,

$$
G_{1 \alpha}^{<}\left(t, t^{\prime}\right)=-T_{1 \alpha} \int d t_{1}\left[G_{1,1}^{R}\left(t, t_{1}\right) g_{\alpha}^{<}\left(t_{1}-t^{\prime}\right)+G_{1,1}^{<}\left(t, t_{1}\right) g_{\alpha}^{A}\left(t_{1}-t^{\prime}\right)\right] .
$$

The functions $g_{\alpha}^{R}\left(t_{1}-t^{\prime}\right), g_{\alpha}^{A}\left(t_{1}-t^{\prime}\right)$ and $g_{\alpha}^{<}\left(t_{1}-t^{\prime}\right)$, with $g_{\alpha}^{A}\left(t-t^{\prime}\right)=\left[g_{\alpha}^{R}\left(t^{\prime}-t\right)\right]^{*}$ are equilibrium Green functions corresponding to the noninteracting system $\alpha$ before the coupling to the ring is performed. In terms of the spectral density $\rho_{\alpha}(\omega)=-2 \operatorname{Im}\left[g_{\alpha}^{R}(\omega)\right]$ and the Fermi function $f_{\alpha}(\omega)=\Theta(\mu-\omega)$ of this system, they read

$$
\begin{aligned}
& g_{\alpha}^{R}\left(t-t^{\prime}\right)=-i \Theta\left(t-t^{\prime}\right) \int \frac{d \omega}{2 \pi} \rho_{\alpha}(\omega) e^{-i \omega\left(t-t^{\prime}\right)}, \\
& g_{\alpha}^{<}\left(t-t^{\prime}\right)=i \int \frac{d \omega}{2 \pi} f_{\alpha}(\omega) \rho_{\alpha}(\omega) e^{-i \omega\left(t-t^{\prime}\right)} .
\end{aligned}
$$

The Green functions $G_{1,1}^{R}\left(t, t^{\prime}\right)$ and $G_{1,1}^{<}\left(t, t^{\prime}\right)$ correspond to the ring coupled to the system $\alpha$. They satisfy the following equations

$$
\begin{aligned}
& G_{1,1}^{R}\left(t, t^{\prime}\right)=g_{1,1}^{R}\left(t, t^{\prime}\right)+\int d t_{1} d t_{2} G_{1,1}^{R}\left(t, t_{1}\right) \Sigma_{1}^{R}\left(t_{1},-t_{2}\right) g_{1,1}^{R}\left(t_{2}, t^{\prime}\right), \\
& G_{1,1}^{<}\left(t, t^{\prime}\right)=\int d t_{1} d t_{2} G_{1,1}^{R}\left(t, t_{1}\right) \Sigma_{1}^{<}\left(t_{1}-t_{2}\right) G_{1,1}^{A}\left(t_{2}, t^{\prime}\right)
\end{aligned}
$$

where $g_{1,1}^{R}\left(t, t^{\prime}\right)$ is the retarded Green function for the uncoupled ring penetrated by the magnetic field. The selfenergies

$$
\begin{aligned}
& \Sigma_{1}^{R}\left(t-t^{\prime}\right)=\left|T_{1 \alpha}\right|^{2} g_{\alpha}^{R}\left(t-t^{\prime}\right), \\
& \Sigma_{1}^{<}\left(t-t^{\prime}\right)=\left|T_{1 \alpha}\right|^{2} g_{\alpha}^{<}\left(t-t^{\prime}\right)
\end{aligned}
$$

account for the corrections due to the escape to the leads 12,13 . This set of equations provide the exact solution for the time-dependent current $J_{1 \alpha}(t)$.

We now introduce one simple model to describe the system $\alpha$. To simplify the numerical procedure, we assume that the ensuing electronic band has a large bandwidth and a constant density of states $\rho_{\alpha}(\omega)=\Gamma$. Thus, $g_{\alpha}^{R}\left(t-t^{\prime}\right) \sim$ $-i \Gamma \delta\left(t-t^{\prime}\right)$ and the following expression for the time-dependent current is obtained

$$
J_{1 \alpha}(t)=\frac{2 e}{\hbar} \sigma \int \frac{d \omega}{\pi} f_{\alpha}(\omega)\left(\operatorname{Im}\left[G_{1,1}^{R}(t, \omega)\right]+\sigma\left|G_{1,1}^{R}(t, \omega)\right|^{2}\right),
$$

where $\sigma=\left|T_{1 \alpha}\right|^{2} \Gamma$, with $\Sigma_{1}^{R}\left(t-t^{\prime}\right)=i \sigma \delta\left(t-t^{\prime}\right)$, while the Fourier transform for the retarded Green function is defined as,

$$
G_{m, n}^{R}(t, \omega)=\int_{-\infty}^{t} d t^{\prime} e^{i(\omega+i \eta)\left(t-t^{\prime}\right)} G_{m, n}^{R}\left(t, t^{\prime}\right),
$$

with $\eta=0^{+}$.

We remark now some salient issues related with expression (9). The function $\rho_{l}(t, \omega)=-2 \operatorname{Im}\left[G_{l, l}^{R}(t, \omega)\right]$ defines a generalized time-dependent spectral function for site $l$, while the function $N_{l}(t, \omega)=2 \sigma\left|G_{l, 1}^{R}(t, \omega)\right|^{2}$ defines the occupation of this site at time $t$, since

$$
N_{l}(t)=-i G_{l l}^{<}(t, t)=\int \frac{d \omega}{2 \pi} f_{\alpha}(\omega) N_{l}(t, \omega),
$$


where $N_{l}(t)=\left\langle c_{l}^{\dagger} c_{l}\right\rangle$ is the mean number of particles at site $l$. In an equilibrium system or in a steady regime where $G_{l, l}^{R}(t, \omega)$ does not have an explicit time dependence, it is easy to prove that $N_{l}(\omega)=\rho_{l}(\omega)$. However, in a non-equilibrium time-dependent regime the local Green function $G_{l, l}^{R}(t, \omega)$ depends explicitly on time and can develop a complex structure as a function of $\omega$. Therefore, the two functions are in general different. This property is crucial for the physical effects we aim to address. In particular, the fact that in a stationary situation these two functions coincide, cause the current $J_{1 \alpha}(t)$ to vanish. Instead, in a non-equilibrium regime, an ac current can flow through the lead. As we shall see, this current exhibits a periodic behavior which is originated by the existence of Bloch oscillations in the ring.

To complete the theoretical description of the system, we define the current flowing through the bond between sites $l$ and $l+1$ along the ring

$$
J_{l, l+1}=\frac{2 e}{\hbar} R e\left[T e^{-i \phi t} G_{l, l+1}^{<}(t, t)\right]
$$

where

$$
G_{l, l+1}^{<}(t, t)=i \int \frac{d \omega}{\pi} \sigma G_{l, 1}^{R}(t, \omega)\left[G_{l+1,1}^{R}(t, \omega)\right]^{*}
$$

with

$$
G_{m, n}^{R}\left(t, t^{\prime}\right)=g_{m, n}^{R}\left(t, t^{\prime}\right)+i \int d t_{1} G_{m, 1}^{R}\left(t, t_{1}\right) \sigma g_{1, n}^{R}\left(t_{1}, t^{\prime}\right)
$$

From the practical point of view, the problem reduces to the calculation of the retarded Green functions $g_{m, n}^{R}\left(t, t^{\prime}\right)$ and $G_{m, n}^{R}\left(t, t^{\prime}\right)$ corresponding the loop penetrated by the magnetic field unconected and coupled to the external reservoir, respectively. This is the subject of Sec. III.

\section{CALCULATION OF THE RETARDED GREEN FUNCTIONS}

\section{A. The metallic ring threaded by a flux $\Phi_{M}(t)=\Phi t$}

We are interested in calculating the retarded Green function $g_{m, n}^{R}\left(t, t^{\prime}\right)$, where $m, n$ label the $m$-th and $n$-th sites of the ring, respectively. The usual procedure to compute this quantity is to take advantage of the translational invariance of the system [17]. In this subsection, we shall discuss an alternative way to obtain exactly $g_{m, n}^{R}\left(t, t^{\prime}\right)$. As we shall see, this route leads to a more transparent physical picture for the relevant energy scales of the problem while it has the additional advantage of being adequate to solve more general problems without translational invariance. We shall see in the next subsection that this procedure also provides a very convenient framework to treat the coupling of the ring with the external lead and reservoir.

The Dyson equation for the retarded Green function leads to the following set of coupled differential equations

$$
-i \hbar \frac{\partial}{\partial t^{\prime}} g_{m, n}^{R}\left(t, t^{\prime}\right)+T e^{i \phi t^{\prime}} g_{m, n+1}^{R}\left(t, t^{\prime}\right)+T e^{-i \phi t^{\prime}} g_{m, n-1}^{R}\left(t, t^{\prime}\right)=\delta\left(t-t^{\prime}\right) \delta_{m, n}
$$

Our strategy is to perform a gauge transformation in order to translate the original problem into an equivalent one where the explicit time dependence appears only in the matrix elements involving the link between the first and the last sites of the ring. By defining the gauge transformation

$$
c_{n}=\exp [i n \phi t] \bar{c}_{n}
$$

the Green function transforms as

$$
g_{m, n}^{R}\left(t, t^{\prime}\right)=\exp \left[i \phi\left(m t-n t^{\prime}\right)\right] \bar{g}_{m, n}^{R}\left(t, t^{\prime}\right) .
$$

From (15), it is found that $\bar{g}_{m, n}^{R}\left(t, t^{\prime}\right)$ satisfies 


$$
\begin{aligned}
& -i \hbar \frac{\partial}{\partial t^{\prime}} \bar{g}_{m, n}^{R}\left(t, t^{\prime}\right)-\hbar \phi n \bar{g}_{m, n}^{R}\left(t, t^{\prime}\right)+T \bar{g}_{m, n+1}^{R}\left(t, t^{\prime}\right)+T \bar{g}_{m, n-1}^{R}\left(t, t^{\prime}\right) \\
& =\delta\left(t, t^{\prime}\right) \delta_{m, n}, \quad n \neq 1, N \\
& -i \hbar \frac{\partial}{\partial t^{\prime}} \bar{g}_{m, 1}^{R}\left(t, t^{\prime}\right)-\hbar \phi \bar{g}_{m, 1}^{R}\left(t, t^{\prime}\right)+T \bar{g}_{m, 2}^{R}\left(t, t^{\prime}\right)+T_{N 1}\left(t^{\prime}\right) \bar{g}_{m, N}^{R}\left(t, t^{\prime}\right) \\
& =\delta\left(t, t^{\prime}\right) \delta_{m, 1}, \quad n=1 \\
& -i \hbar \frac{\partial}{\partial t^{\prime}} \bar{g}_{m, N}^{R}\left(t, t^{\prime}\right)-\hbar \frac{\Phi}{\Phi_{0}} \bar{g}_{m, N}^{R}\left(t, t^{\prime}\right)+T_{1 N}\left(t^{\prime}\right) \bar{g}_{m, 1}^{R}\left(t, t^{\prime}\right)+T \bar{g}_{m, N-1}^{R}\left(t, t^{\prime}\right) \\
& =\delta\left(t, t^{\prime}\right) \delta_{m, N} . \quad n=N,
\end{aligned}
$$

where the only matrix elements that exhibit an explicit time dependence are

$$
T_{1 N}(t)=\exp \left[i \frac{\Phi}{\Phi_{0}} t\right] T
$$

with $\left[T_{N 1}(t)\right]^{*}=T_{1 N}(t)$.

The above set can be interpreted as the Dyson equation for the retarded Green function corresponding to the problem of a chain with $N$ sites of tight-binding electrons in the presence of a dc voltage

$$
V_{l}=\hbar l \phi .
$$

The chain is closed at its ends by a time dependent link $T_{1 N}(t)$. We formally treat this system as indicated in the scheme of Fig. 2, by separating the ensuing hamiltonian in an "unperturbed" part that describes an open tight-binding chain,

$$
\bar{H}^{0}=-T \sum_{l=1}^{N-1}\left(\bar{c}_{l}^{\dagger} \bar{c}_{l+1}+h . c\right)+\sum_{l=1}^{N} V_{l} \bar{c}_{l}^{\dagger} \bar{c}_{l},
$$

and a time-dependent "perturbation"

$$
\bar{H}_{1 N}(t)=-T_{1 N}(t) \bar{c}_{1}^{\dagger} \bar{c}_{N}-T_{N 1}(t) \bar{c}_{N}^{\dagger} \bar{c}_{1},
$$

which describes a time-dependent hopping between the first and the last sites of the chain. Within this picture, the integral form for the Dyson equation reads

$$
\begin{aligned}
\bar{g}_{m, n}^{R}\left(t, t^{\prime}\right) & =g_{m, n}^{0}\left(t, t^{\prime}\right)-\int_{t^{\prime}}^{t} d t_{1} \bar{g}_{m, N}^{R}\left(t, t_{1}\right) T_{N 1}\left(t_{1}\right) g_{1, n}^{0}\left(t_{1}, t^{\prime}\right) \\
& -\int_{t^{\prime}}^{t} d t_{1} \bar{g}_{m, 1}^{R}\left(t, t_{1}\right) T_{1 N}\left(t_{1}\right) g_{N, n}^{0}\left(t_{1}, t^{\prime}\right),
\end{aligned}
$$

where the matrix elements $T_{N 1}(t)$ and $T_{1 N}(t)$ define time-dependent instantaneous self-energies. The "unperturbed" Green function $g_{m, n}^{0}\left(t, t^{\prime}\right) \equiv g_{m, n}^{0}\left(t-t^{\prime}\right)$ is an equilibrium Green function corresponding to the hamiltonian $\bar{H}_{0}$. Although there is no trivial analytical expression for $g_{m, n}^{0}\left(t-t^{\prime}\right)$, it can be easily obtained in a finite-size system from the numerical solution of hamiltonian (21). In terms of the eigenvalues $E_{\nu}$ and eigenvectors $|\nu\rangle=\sum_{l} A_{l}^{\nu}|l\rangle$ of $\bar{H}_{0}$, it can be written as

$$
g_{m, n}^{0}\left(t-t^{\prime}\right)=-i \Theta\left(t-t^{\prime}\right) \sum_{\nu=1}^{N} A_{m}^{\nu} A_{n}^{\nu} \exp \left[-\frac{i}{\hbar} E_{\nu}\left(t-t^{\prime}\right)\right] .
$$

After performing in Eqs. (23) the Fourier transform defined in (10), we obtain for $n=1$

$$
\begin{gathered}
\bar{g}_{m, 1}^{R}(t, \omega)+\bar{g}_{m, N}^{R}\left(t, \omega+\frac{\Phi}{\Phi_{0}}\right) T_{N 1}(t) g_{1,1}^{0}(\omega)+\bar{g}_{m, 1}^{R}\left(t, \omega-\frac{\Phi}{\Phi_{0}}\right) T_{1 N}(t) g_{N, 1}^{0}(\omega)=g_{m, 1}^{0}(\omega) \\
\bar{g}_{m, N}^{R}(t, \omega)+\bar{g}_{m, N}^{R}\left(t, \omega+\frac{\Phi}{\Phi_{0}}\right) T_{N 1}(t) g_{1, N}^{0}(\omega)+\bar{g}_{m, 1}^{R}\left(t, \omega-\frac{\Phi}{\Phi_{0}}\right) T_{1 N}(t) g_{N, N}^{0}(\omega)=g_{m, N}^{0}(\omega) .
\end{gathered}
$$

For each time $t$, the solution of the above set of linear equations provides the complete exact solution of the problem. The Green function for arbitrary $m, n$ is obtained from 


$$
\bar{g}_{m, n}^{R}(t, \omega)=g_{m, n}^{0}(t, \omega)-\bar{g}_{m, N}^{R}\left(t, \omega+\frac{\Phi}{\Phi_{0}}\right) T_{N 1}(t) g_{1, n}^{0}(\omega)-\bar{g}_{m, 1}^{R}\left(t, \omega-\frac{\Phi}{\Phi_{0}}\right) T_{1 N}(t) g_{N, n}^{0}(\omega),
$$

while the Fourier transform of the retarded Green function of the original problem is

$$
g_{m, n}(t, \omega)=\exp [i \phi(m-n) t] \bar{g}_{m, n}^{R}(t, \omega-n \phi) .
$$

\section{B. The ring coupled to the particle reservoir}

We now develop a computational strategy to obtain the retarded Green function $G_{m, n}^{R}\left(t, t^{\prime}\right)$ for the coupled system. The differential form of the Dyson equation (14) for this Green function reads

$$
\begin{aligned}
& -i \hbar \frac{\partial}{\partial t^{\prime}} G_{m, n}^{R}\left(t, t^{\prime}\right)-i \sigma \delta_{n, 1} G_{m, n}^{R}+T e^{-i \phi t^{\prime}} G_{m, n+1}^{R}\left(t, t^{\prime}\right) \\
& +T e^{i \phi t^{\prime}} G_{m, n-1}^{R}\left(t, t^{\prime}\right)=\delta_{m, n} \delta\left(t-t^{\prime}\right) .
\end{aligned}
$$

As in the previous subsection, we perform the gauge transformation (16) in order to obtain a set of equations with a reduced number of time-dependent matrix elements. The Green function transforms as

$$
G_{m, n}^{R}\left(t, t^{\prime}\right)=\exp \left[i \phi\left(m t-n t^{\prime}\right)\right] \bar{G}_{m, n}^{R}\left(t, t^{\prime}\right)
$$

and $\bar{G}_{m, n}^{R}\left(t, t^{\prime}\right)$ satisfies the following equations

$$
\begin{aligned}
& -i \hbar \frac{\partial}{\partial t^{\prime}} \bar{G}_{m, n}^{R}\left(t, t^{\prime}\right)-\hbar \phi n \bar{G}_{m, n}^{R}\left(t, t^{\prime}\right)+T \bar{G}_{m, n+1}^{R}\left(t, t^{\prime}\right)+T \bar{G}_{m, n-1}^{R}\left(t, t^{\prime}\right) \\
& =\delta_{m, n} \delta\left(t-t^{\prime}\right) \quad n \neq 1, N \\
& -i \hbar \frac{\partial}{\partial t^{\prime}} \bar{G}_{m, 1}^{R}\left(t, t^{\prime}\right)-\hbar \phi \bar{G}_{m, 1}^{R}\left(t, t^{\prime}\right)-i \sigma \bar{G}_{m, 1}^{R}\left(t, t^{\prime}\right)+T \bar{G}_{m, 2}^{R}\left(t, t^{\prime}\right)+T_{N, 1}\left(t^{\prime}\right) \bar{G}_{m, N}^{R}\left(t, t^{\prime}\right) \\
& =\delta_{m, 1} \delta\left(t-t^{\prime}\right) \\
& -i \hbar \frac{\partial}{\partial t^{\prime}} \bar{G}_{m, N}^{R}\left(t, t^{\prime}\right)-\hbar \frac{\Phi}{\Phi_{0}} \bar{G}_{m, N}^{R}\left(t, t^{\prime}\right)+T_{1, N}\left(t^{\prime}\right) \bar{G}_{m, 1}^{R}\left(t, t^{\prime}\right)+T \bar{G}_{m, N-1}^{R}\left(t, t^{\prime}\right) \\
& =\delta_{m, N} \delta\left(t-t^{\prime}\right)
\end{aligned}
$$

The above set is formally identical to the Dyson equation of the problem illustrated in Fig. 3. This consists in an open tight-binding chain of $N-1$ sites labeled by $l=2, \ldots, N$ with potentials $V_{l}$ (see (20)), plus an additional site labeled by $l=1$ (indicated with a square in Fig. 3). The latter represents a site of the ring coupled to the lead and reservoir. It has a local potential $V_{1}$ and a self-energy correction $i \sigma$ due to the coupling to the system $\alpha$. This site is connected to one end of the chain through a hopping $T$ and to the other end, through the time-dependent hopping $T_{1 N}(t)(19)$. The integral form of the Dyson equation results,

$$
\begin{aligned}
\bar{G}_{m, n}^{R}\left(t, t^{\prime}\right)= & g_{m, n}^{0}\left(t, t^{\prime}\right)-\int d \omega d t_{1} \bar{G}_{m, N}^{R}\left(t, t_{1}\right) T_{N 1}\left(t_{1}\right) g_{1, n}^{0}\left(t_{1}, t^{\prime}\right)- \\
& \int d \omega d t_{1} \bar{G}_{m, 1}^{R}\left(t, t_{1}\right) T_{1 N}\left(t_{1}\right) g_{N, n}^{0}\left(t_{1}, t^{\prime}\right)-T \int d t_{1} \bar{G}_{m, 1}^{R}\left(t, t_{1}\right) g_{2, n}^{0}\left(t_{1}, t^{\prime}\right)- \\
& T \int d t_{1} \bar{G}_{m, 2}^{R}\left(t, t_{1}\right) g_{1, n}^{0}\left(t_{1}, t^{\prime}\right),
\end{aligned}
$$

which by recourse to the Fourier transform (10) leads to the following set

$$
\begin{aligned}
& \bar{G}_{m, 1}^{R}(t, \omega)=g_{m, 1}^{0}(\omega)-T e^{-i \frac{\Phi}{\Phi_{0}} t} \bar{G}_{m, N}^{R}\left(t, \omega+\hbar \frac{\Phi}{\Phi_{0}}\right) g_{1,1}^{0}(\omega)-T \bar{G}_{m, 2}^{R}(t, \omega) g_{1,1}^{0}(\omega) \\
& \bar{G}_{m, N}^{R}(t, \omega)=g_{m, N}^{0}(\omega)-T e^{i \frac{\Phi}{\Phi_{0}} t} \bar{G}_{m, 1}^{R}\left(t, \omega-\hbar \frac{\Phi}{\Phi_{0}}\right) g_{N, N}^{0}(\omega)-T \bar{G}_{m, 1}^{R}(t, \omega) g_{2, N}^{0}(\omega) \\
& \bar{G}_{m, 2}^{R}(t, \omega)=g_{m, 2}^{0}(\omega)-T e^{i \frac{\Phi}{\Phi_{0}} t} \bar{G}_{m, 1}^{R}\left(t, \omega-\hbar \frac{\Phi}{\Phi_{0}}\right) g_{N, 2}^{0}(\omega)-T \bar{G}_{m, 1}^{R}(t, \omega) g_{2,2}^{0}(\omega),
\end{aligned}
$$


where $g_{m, n}^{0}(\omega)$ with $m, n=2, \ldots, N$ is the Green function of the chain formed with sites $2, \ldots, N$ with a bias $V_{l}$, while $g_{1,1}^{0}=1 /(\omega-\phi+i \sigma)$ is the Green function for the remaining site in interaction with system $\alpha$.

After some rather simple algebra, it is easy to see that Eqs. (32) can be reduced to the set (25) by replacing in the latter $\bar{g}_{m, n}^{R}(t, \omega)$ by $\bar{G}_{m, n}^{R}(t, \omega)$ and $g_{m, n}^{0}(t, \omega)$ by $G_{m, n}^{0}(t, \omega)$, being

$$
G_{m, n}^{0}(\omega)=g_{m, n}^{0}(\omega)-T G_{m, 1}^{0}(\omega) g_{2, n}^{0}-T G_{m, 2}^{0}(\omega) g_{1, n}^{0}(\omega)
$$

A scheme of the underlying physical processes related with this equation is given in Fig. 3b. It corresponds to the coupling of the open chain with the site $l=1$ by means of a single hopping element $T$. Equations (25) correspond to the closing of the resulting $N$-site chain by means of a time-dependent hopping process (22) between its ends.

In conclusion, the exact Green function for the loop penetrated by the time-dependent magnetic field, as an isolated system or as a system coupled to an external particle reservoir, is given by the solution of Eqs. (25), provided that a suitable expression for the "unpperturbed" Green function, $g_{m, n}^{0}(t, \omega)$ or $G_{m, n}^{0}(t, \omega)$ correspondingly, is given. We must emphasize that although in both cases the final set of equations is formally identical, physical processes of different nature are enclosed in the "unperturbed" Green function. In particular in the case considered in this subsection, the effect of the lead represented by the self-energy $i \sigma$ introduces inelastic scattering events that produce loss of coherence in the propagation of the wave function through the ring and dissipation.

The imaginary part of the local Green function defines a generalized time-dependent density of states $\rho_{l}(t, \omega)=$ $-2 \operatorname{Im}\left[G_{l, l}^{R}(t, \omega)\right]$, in terms of which the local Green function can be written as

$$
G_{l, l}^{R}(t, \omega)=\int \frac{d \omega}{2 \pi} \frac{\rho_{l}(t, \omega)}{\omega-\omega^{\prime}+i \eta} .
$$

This quantity is in general expected to be a periodic function on time, as suggested by the structure of the set (25). However, we shall later see that in some situations it can also become independent of time.

\section{RESULTS}

\section{A. Dynamical regimes}

We first consider the case introduced in Section IIIA, which corresponds to the ring under the influence of the time-varying magnetic flux, but disconnected from the external wire and particle reservoir. Our aim is to understand first the dynamical behavior of this system, free from the effects of dissipation introduced by the coupling to the external reservoir.

An important issue is that if $g_{1, N}^{0}(\omega)=g_{N, 1}^{0}(\omega)=0$, the equations in (25) can be decoupled. In that case, the solution becomes stationary, i.e. does not have any dependence on time and reads

$$
\bar{g}_{m, n}^{R}(t, \omega) \equiv \bar{g}_{m, n}^{R}(\omega)=\frac{g_{m, n}^{0}(\omega)}{1-T^{2} g_{11}^{0}(\omega) g_{N N}^{0}\left(\omega+\Phi / \Phi_{0}\right)} .
$$

The latter property implies that the local Green functions of the original problem $g_{l, l}(t, \omega)$ become independent of time, while the non-local ones exhibit a trivial time dependence introduced by the gauge transformation (27).

In order to find out the physical conditions in which this stationary solution is expected, we study the behavior of $g_{1, N}^{0}(\omega)$. The relevant parameters are the magnitude $\hbar \Phi / \Phi_{0}$ relative to the bandwidth of the tight-binding chain $W=4 T$ and the size of the system $N$. We numerically diagonalized the hamiltonian (21) for different ratios $\hbar \Phi / \Phi_{0} W$ and studied systems with sizes up to $N=800$ sites. Typical curves for the imaginary part

$$
\operatorname{Im}\left[g_{1, N}^{0}(\omega)\right]=-\pi \sum_{\nu=1}^{N} A_{1}^{\nu} A_{N}^{\nu} \delta\left(\omega-E_{\nu}\right) .
$$

for a chain with $N=20$ sites are shown in Fig. 4. It is clear that for all $\omega$, the magnitude of this quantity is significantly larger in the cases where $\hbar \Phi / \Phi_{0}<W$.

To be able to perform a more quantitative analysis of the behavior of $g_{1, N}^{0}(\omega)$ as a function of $N$, we consider the function $g_{\max }(N)$, which is defined as the maximum value achieved by $\left|\operatorname{Im}\left[g_{1 L}^{0}(\omega)\right]\right|$ for a given size $N$. Plots of $g_{\max }(N)$ as a function of $N$ reveal that there is a change in the scaling of this quantity as $\hbar \Phi / \Phi_{0} W$ varies. The case $\hbar \Phi \leq \Phi_{0} W$ is illustrated in Fig. 5. We found that the scaling law is 


$$
g_{\max }(N) \propto \frac{1}{N^{\gamma}}, \quad \hbar \Phi / \Phi_{0} \leq W .
$$

The data of Fig. 5 can be adequately fitted with $\gamma \sim 1$ for $\hbar \Phi / \Phi_{0} W=0,0.25,0.5,0.75$ and $\gamma \sim 1.4$ for $\hbar \Phi / \Phi_{0} W=1$. The opposite situation, where $\hbar \Phi / \Phi_{0}>W$ is shown in Fig. 6 . We found that in this case, the scaling law is

$$
g_{\max }(N) \propto e^{-\frac{N}{L_{\alpha}}}, \quad \hbar \Phi / \Phi_{0}>W .
$$

By fitting the numerical data it is obtained $L_{\alpha} \sim 8$ for $\hbar \Phi / \Phi_{0} W=1.25$ and $L_{\alpha} \sim 4$ for $\hbar \Phi / \Phi_{0} W=1.5$.

The existence of two kinds of behaviors in the scaling of $g_{1, N}^{0}(\omega)$ has a rather simple explanation. We recall that this Green function corresponds to a system of electrons in an open tight-binding chain with a bias $\hbar \Phi / \Phi_{0}$ between its edges. The different scaling laws result from a concurrence between the kinetic energy of the electrons in the chain and the strength of the bias. In fact, the Green function $g_{1, N}^{0}\left(t-t^{\prime}\right)$ is by definition related with overlaps of the form $\left\langle\Psi_{1}(t) \mid \Psi_{L}^{\prime}\left(t^{\prime}\right)\right\rangle$, where $\left|\Psi_{l}(t)\right\rangle$ and $\left|\Psi_{l}^{\prime}(t)\right\rangle$ are the wave functions of the system when an electron is created and destroyed, respectively, in site $l$ at time $t$. This quantity is thus a measure for the ability of an electron to move from the first to the last site of the chain in a time $t-t^{\prime}$. In the free chain, the probability of such a movement decreases as $1 / N$ as the size of the system increases, independently of how large the time it takes is assumed to be. Our numerical analysis indicates that this is also the case in the presence of a bias, provided that the strength of the bias is not larger than the bandwidth associated with the kinetic energy of the electrons. As the bias increases and overcomes the value of the bandwidth, this probability becomes smaller and decreases exponentially with the size of the system.

Therefore, for magnetic fields varying at a rate such that $\hbar \Phi / \Phi_{0} \leq W$, the Green functions $\bar{g}_{m, n}^{R}(t, \omega)$ tend to the steady solution (35) as $N^{-\gamma}$ when the size of the system increases. Hence, for finite size systems, time dependent features are clearly observable. As a function of time, the functions $\bar{g}_{m, n}^{R}(t, \omega)$ oscillate with the period $\tau=2 \pi \Phi_{0} / \Phi$. The latter being the period of the Bloch oscillations due to the induced electric field of magnitude $F=\Phi / c L$. Instead, for rapid variations of the magnetic field such that $\hbar \Phi / \Phi_{0}>W$, the Green functions tend to (35) exponentially fast as the length of the circumference increases. As a consequence, within this regime, time dependent features can be observed only in very small rings of lengths smaller than $L_{\alpha}$.

We close this subsection with some comments about the behavior introduced by inelastic scattering processes in the dynamical behavior above mentioned. We have shown in section IIIB that the Green function for the coupled ring is also given by the solution of Eqs. (25) with the replacement of $g_{m, n}^{0}(\omega)$ by the function $G_{m, n}^{0}(\omega)$ which contains the corrections due to the escape to the leads. Thus, the equations in 25) are decoupled and a stationary solution

$$
\bar{G}_{m, n}^{R}(\omega) \equiv \bar{G}_{m, n}^{R}(\omega)=\frac{G_{m, n}^{0}(\omega)}{1-T^{2} G_{11}^{0}(\omega) G_{N N}^{0}\left(\omega+\Phi / \Phi_{0}\right)} .
$$

exists also in this case when the function $G_{1 L}^{0}(\omega)$ becomes vanishingly small. As before, this quantity is related with the probability of an electron to travel from the first to the last sites of the chain. In this case, the new ingredient is the existence of a typical inelastic scattering length $L_{i n}$ beyond which the coherence in the wave packet propagation is loss. The latter is related to the imaginary part of the self-energy through $\sigma \propto 1 / L_{i n}$ which generates damping and produces an exponential decay $\sim \exp \left[-\alpha L / L_{i n}\right]$ in the Green functions $G_{m, n}^{0}(\omega)$ [15]. Therefore, for rings with circumferences $L<L_{i n}$ the same two dynamical regimes defined for the disconnected ring can be distinguished. Instead, as soon as $L>L_{i n}$, inelastic scattering due to the presence of the external reservoir dominate the physical behavior of the system, the Green functions coincide with the steady solution (35) and no time dependent features are observed. An example of significant time-dependent behavior is shown in Fig. 7 where typical shapes of the local generalized density of states $\rho_{1}(t, \omega)$ for a ring of $N=20$ sites with $\sigma=0.025 \mathrm{~W}$ at different times are depicted. As a function of $\omega, \rho_{1}(t, \omega)$ can be either positive or negative as a consequence of the far from equilibrium nature of this regime. The sum rule

$$
\int \frac{d \omega}{2 \pi} \rho_{1}(t, \omega)=1
$$

is, however, satisfied, justifying the name of density of states.

\section{B. The current through the lead}

Although a classical textbook analysis indicates that the current through the single lead coupling the ring to the particle reservoir should vanish, within the time-dependent regime defined in the previous subsection, there is an ac contribution which could be measured in a real experiment. 
The time-dependent regime is characterized by an imaginary part of the self energy $\sigma$ leading to an inelastic scattering length $L_{i n}$ larger than the size of the ring and a variation of the magnetic field satisfying the condition $\hbar \Phi / \Phi_{0} l e q W$. The propagation of the wave packet maintains the coherence, the Green functions $g_{1,1}(t, \omega)$ and $G_{1,1}(t, \omega)$ oscillate with the period $\tau=2 \pi \Phi_{0} / \Phi$ of Bloch oscillations driven by the induced electric field, and so does the current through the lead. Examples for a ring with $N=20$ sites are shown in Fig. 8 where $J_{1 \alpha}(t)$, obtained from (9), is shown for two different values of $\Phi$. The imaginary part of the self-energy is taken to be $\sigma=0.2 W$. The physical picture that emerges is that the induced electric field produces dramatic changes in the generalized time-dependent density of states in the ring $\rho_{1}(t, \omega)$. This quantity is periodic in time and eventhough it satisfies the sum rule (40), it can achieve positive as well as negative values as a function of $\omega$ (see Fig. 7). In particular, this function does not satisfy the property of being equal to the occupation function $N_{1}(t, \omega)$. As a consequence the integral (9) does not vanish in general but displays oscillations with zero mean value as a function of time. A somehow similar phenomenon in the context of optical properties of semiconductors is the so called Franz-Keldysh effect [16]. In that case, the changes in the density of states due to the influence of time-dependent fields cause the absorption coefficient to become finite for photon energies bellow the band-edge.

Interestingly, due to the symmetry of the spectrum (see Fig. 7) the current $J_{1 \alpha}(t)$ is an antisymmetric function of the chemical potential $\mu$. This property is due to the particular geometry of the system we are considering. Any spectral asymmetry, introduced, for example, by the presence of barriers and/or disorder in the ring should remove this feature.

The effect of inelastic scattering is illustrated in Fig. 9, where $J_{1 \alpha}$ is shown for several values of $\sigma$. Inelastic scattering introduces damping and $L_{i n}$ decreases as $\sigma$ increases. In terms of the spectrum, the peaks of $\rho_{1}(t, \omega)$ get wider and, for very large $\sigma$, the time dependent features tend to be wiped out. On the other hand, very weak inelastic scattering, which occurs for example for a very small value of $T_{1 \alpha}$, tends to forbid the quantum tunneling between the ring and the reservoir. Hence, the amplitude for the oscillations of $J_{1 \alpha}$ is vanishingly small for small $\sigma$, tends to decrease for very large $\sigma$ and attains a maximum in between.

\section{The current along the ring}

The resistive effect caused by inelastic scattering in metallic loops was studied some time ago by Landauer and Büttiker [9], who proposed a phenomenological equation of motion to analyze the relaxation processes in a ring penetrated by a time-dependent magnetic flux. A dc component in the electric current along the ring was found to increase as inelastic scattering is introduced and to decrease as inelastic scattering becomes sufficiently intense. This analysis was later supplemented by Büttiker [10], who proposed a single lead connecting the ring with an external reservoir like in Fig. 1, as a simple device to generate a resistance in the metallic loop.

In this section we show that the theoretical approach adopted in the present work is also appropriate to investigate the effect of inelastic scattering in the current along the ring. Results for the current (12) through the bond between the sites $l=1,2$ of the ring are shown in Fig. 10 for a system with $N=20$ sites. We consider parameters within the time-dependent regime. The imaginary part of the self-energy is assumed to be $\sigma=0.2 \mathrm{~W}$. As a function of time, the current $J_{12}(t)$ displays oscillations with the period $\tau=2 \pi \Phi_{0} / \Phi$ of Bloch oscillations. Note that the factor $e^{-i \phi t}$ in (12) exactly cancels the time-dependent exponential introduced by the gauge transformation (27). Thus, the oscillations of the current $J_{l, l+1}$ result as a consequence of the time-dependent behavior of the Green functions $\bar{G}_{m, n}^{R}(t, \omega)$ obtained from the solution of Eqs. (25) and disappear in the steady regime. The remarkable feature is the existence of a dc component in the current, which depends on the chemical potential of the reservoir, as well as on the rate $\hbar \Phi / \Phi_{0}$ for fixed $\sigma$. The effect of $\sigma$ on the magnitude of the dc component is shown in Fig. 11, where it is observed the behavior predicted in Ref [9], namely the magnitude of this component is vanishes for small as well as for very large $\sigma$ and has a maximum for intermediate strength of dissipation.

To close this section, we show the behavior of the mean occupation number $N_{l}(t)$ along the ring. The latter is calculated from (11) for a ring with $N=20$ sites. Results are shown in Fig. 12 for some values of $l$. As a function of time it displays oscillations with period $\tau$ around the mean value

$$
\left\langle N_{l}\right\rangle=\frac{1}{\tau} \int d t N_{l}(t)
$$

that depends strongly on the position $l$, as shown in Fig. 13. The behavior of $\left\langle N_{l}\right\rangle$ is a measure of the effective mean potential along the chain. The latter exhibits a significant drop near the site $l=1$ where the lead is connected, which becomes more abrupt as the strength of the inelastic scattering introduced by $\sigma$ increases. 


\section{SUMMARY AND CONCLUSIONS}

We have studied the transport properties of a ring threaded by a magnetic flux with a linear dependence on time $\Phi_{M}(t)=\Phi t$. We considered an experimental setup to investigate the transport properties of this system, which consists in a single lead connecting the ring to an external particle reservoir. We employed a theoretical approach based in Baym-Kadanoff-Keldysh non-equilibrium Green functions. We derived a system of linear equations to calculate in real space the retarded Green function of the disconnected ring as well as for the ring coupled to the particle reservoir.

This method provides a transparent picture for the different energy scales governing the dynamics of the system. In particular, we identified two kind of behaviors in the Green functions of the uncoupled ring, depending on the ratio $\hbar \Phi / \Phi_{0} W$. For magnetic fields varying at a rate such that $\hbar \Phi / \Phi_{0} \leq W$, the time dependent features are important in the behavior of the Green functions of finite rings and tend to disappear as $N^{-\gamma}$ when the size of the system increases. Instead, for $\hbar \Phi / \Phi_{0}>W$, the Green functions tend exponentially fast to a static solution as the size of the system increases. These two kinds of behaviors manifest themselves in the transport properties of the system when the inelastic scattering length is larger than the perimeter of the ring. Within the time-dependent regime, the current through the lead as well as the current along the ring and the charge inside it, display oscillations with the period $\tau=2 \pi \Phi_{0} / \Phi$ of Bloch oscillations driven by the induced electric field. In the situation of a rapid magnetic field variation satisfying $\hbar \Phi / \Phi_{0}>W$, no current is measured in the lead and no oscillatory behavior is observed except for extremely small rings. We stress that this effect has its grounds in the concurrence between the kinetic energy of the electrons and the strength of the dc voltage due to the induced electric field, being independent of the loss of coherence introduced by inelastic scattering.

We finally analyzed the effect of inelastic scattering caused by the coupling with the external reservoir in the timedependent transport. We found that the amplitude of the oscillations of the current through the lead as well as the dc component of the current along the ring, tend to vanish in the two limiting situations of very weak and very strong inelastic scattering, attaining a maximum for intermediate strengths.

On the technical side, we find that the present approach provides an useful theoretical tool to investigate the effects of time dependent magnetic fluxes and induced electric fields in other interesting systems of larger complexity, like metallic loops with disorder, rings with inserted barriers and quantum dots and one dimensional systems with electronic correlations.

\section{ACKNOWLEDGEMENTS.}

L. A. is supported by CONICET and Fundación Antorchas. Support from the AvH foundation at the first stage of this work is acknowledged. The author thanks G. Chiappe and H. Pastawski for stimulating discussions.

[1] V. Chandrasekar et. al, Phys. Rev. Lett 67, 3578 (1991).

[2] Y. Gefen, Y. Imry and M. Ya. Azbel, Phys. Rev. Lett. 52129 (1984).

[3] F. Bloch, Z. Phys. 52, 555 (1928).

[4] C. Waschke et. al, Phys. Rev. Lett. 70, 3318 (1993).

[5] J. Rotvig, A. P. Jauho, and H. Smith, Phys. Rev. Lett. 74, 1831 (1995).

[6] M. Holthaus, G. Ristow and D. Hone, Phys. Rev. Lett. 75, 3914 (1995).

[7] V. Grecchi and A. Sacchetti, Phys. Rev. Lett. 78, 4474 (1997).

[8] M. Büttiker, Y. Imry and R. Landauer, Phys. Lett. A 96A, 365 (1983).

[9] R. Landauer and M. Büttiker, Phys. Rev. Lett. 542049 (1985).

[10] M. Büttiker, Phys. Rev. B 32, R1846 (1985).

[11] A. Yacobi, M. Heiblum, D. Mahalu and H. Shtrikman, Phys. Rev. Lett 74, 4047 (1995).

[12] H. M. Pastawski, Phys. Rev. B 46, 4053 (1992).

[13] A. P.Jauho, N. Wingreen and Y. Meir, Phys. Rev. B 50, 5528 (1994).

[14] Y Cheung, E. Riedel and Y. Gefen, Phys. Rev. Lett. 62, 587 (1989).

[15] J. L. D'Amato and H. M. Pastawski, Phys. Rev. B 41, 7411 (1990).

[16] A. P. Jauho and K. Johnsen, Phys. Rev. Lett. 764576 (1996). 
FIG. 1. (a) Scheme of the experimental setup to study the charge transport in a metallic ring threaded by a time-dependent magnetic field. (b) Theoretical model for the device in (a).

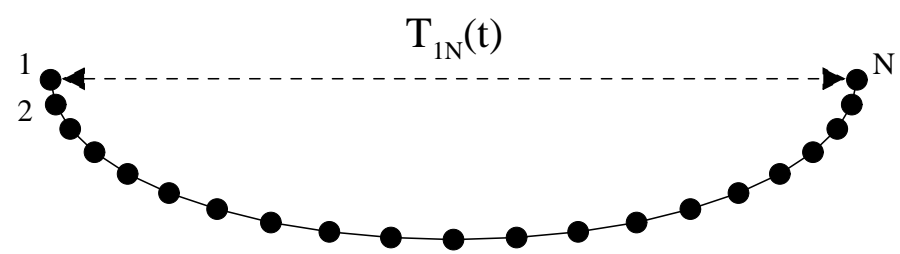

FIG. 2. Scheme of the physical processes involved in the Dyson equation for the retarded Green function (23).

[17] The analytical expresion for the Fourier transform is $g_{k}^{R}\left(t, t^{\prime}\right)=-i \Theta\left(t-t^{\prime}\right) \int_{t}^{t^{\prime}} d s \exp \left[-i \epsilon_{k}(s)\right]$, where $\epsilon_{k}(s)=-2 T \cos (k+$ $\phi s)$, with $k=2 n \pi / N, n=0, \ldots, N-1$. 

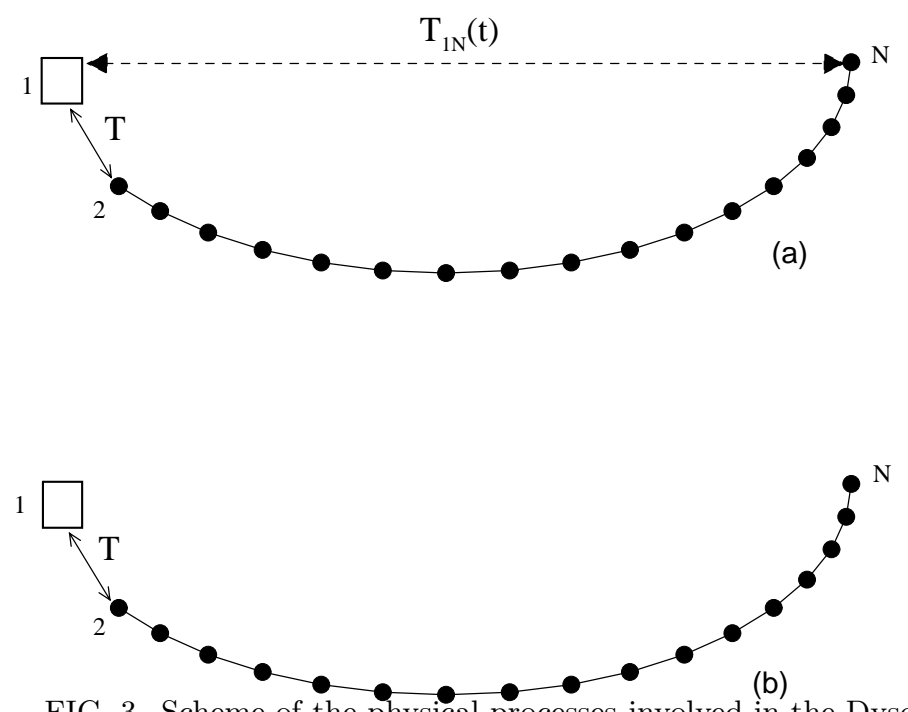

FIG. 3. Scheme of the physical processes involved in the Dyson equation for the retarded Green function. Figs. (a) and (b) correspond to Eqs. (32) and (33), respectively.
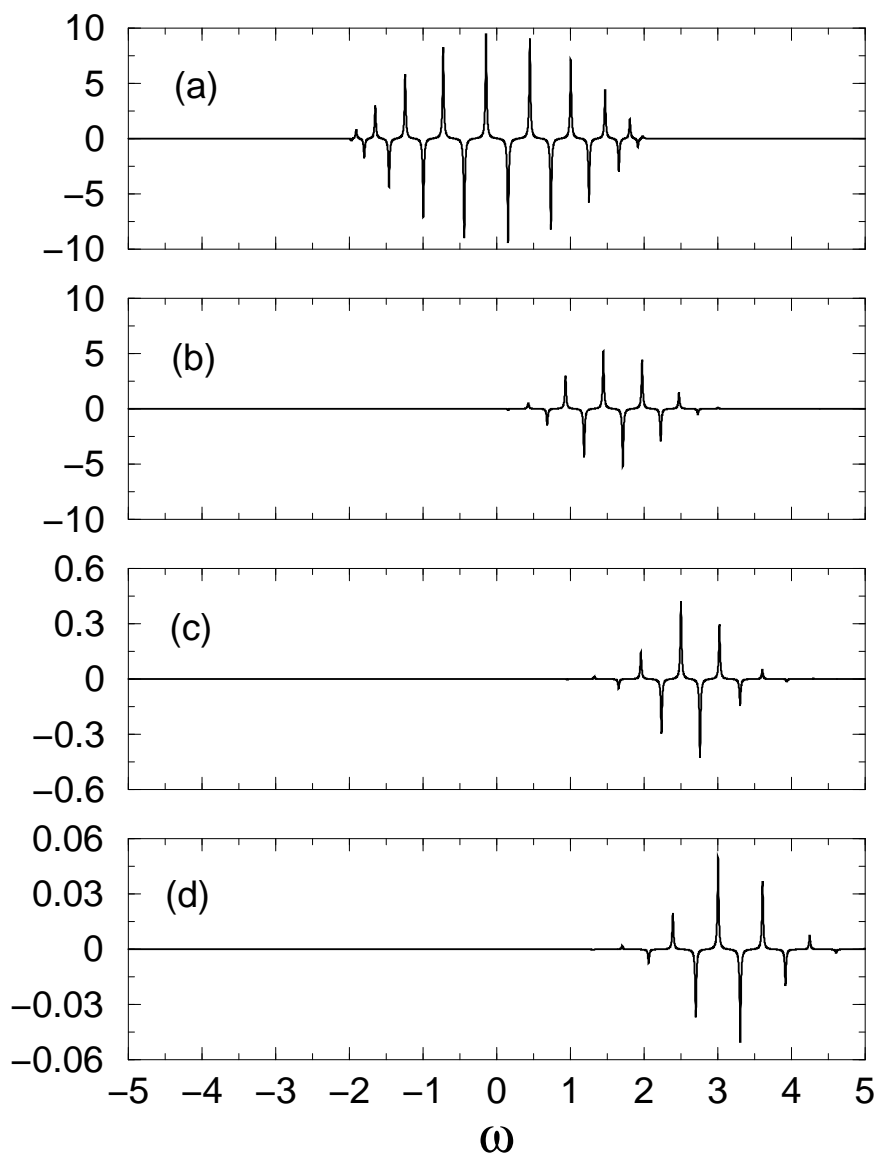

FIG. 4. Imaginary part of the Green function $g_{1 N}^{0}(\omega)$ for a system of $N=20$ sites and magnetic fluxes varying at rates $\hbar \Phi / \Phi_{0}=0,0.75 \mathrm{~W}, 1.25 \mathrm{~W}, 1.5 \mathrm{~W}$ for (a), (b), (c) and (d), respectively. 


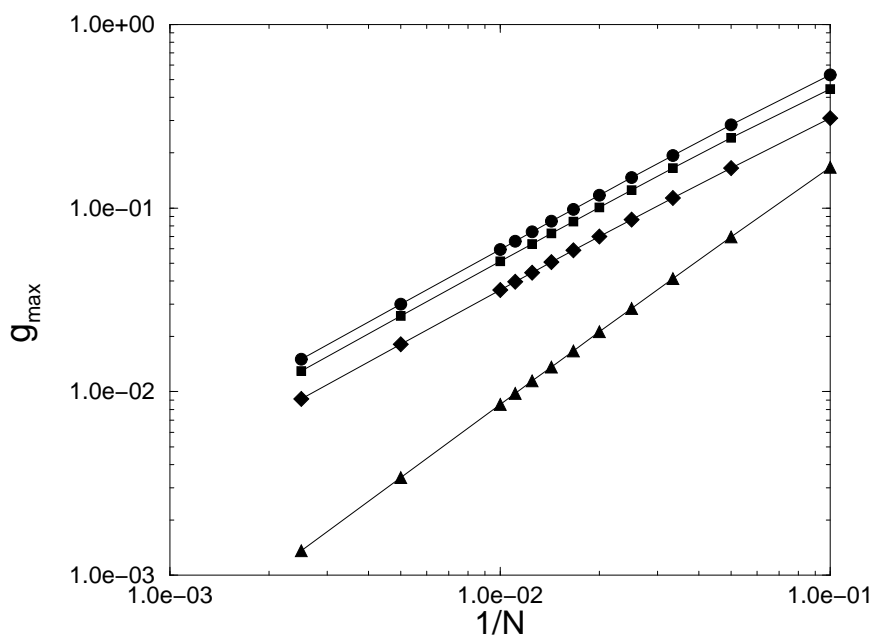

FIG. 5. $g_{\max }$ as a function of $1 / N$. Circles, squares, diamonds and triangles correspond to $\hbar \Phi / \Phi_{0}=0.25 W, 0.33 W, 0.5 W, W$, respectively.

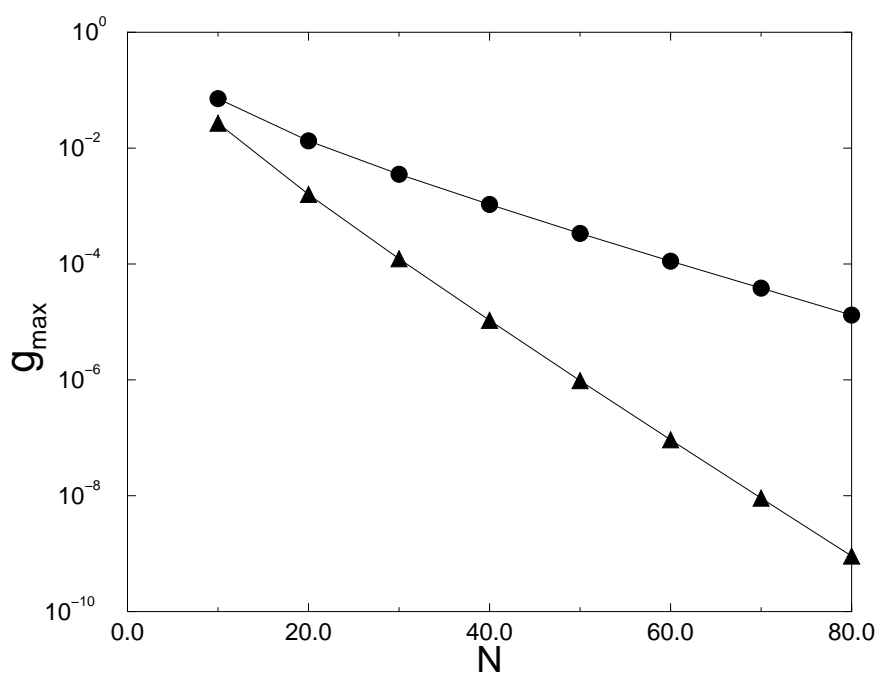

FIG. 6. $g_{\max }$ as a function of $N$. Circles and squares correspond to $\hbar \Phi / \Phi_{0}=1.25 W, 1.5 W$, respectively. 


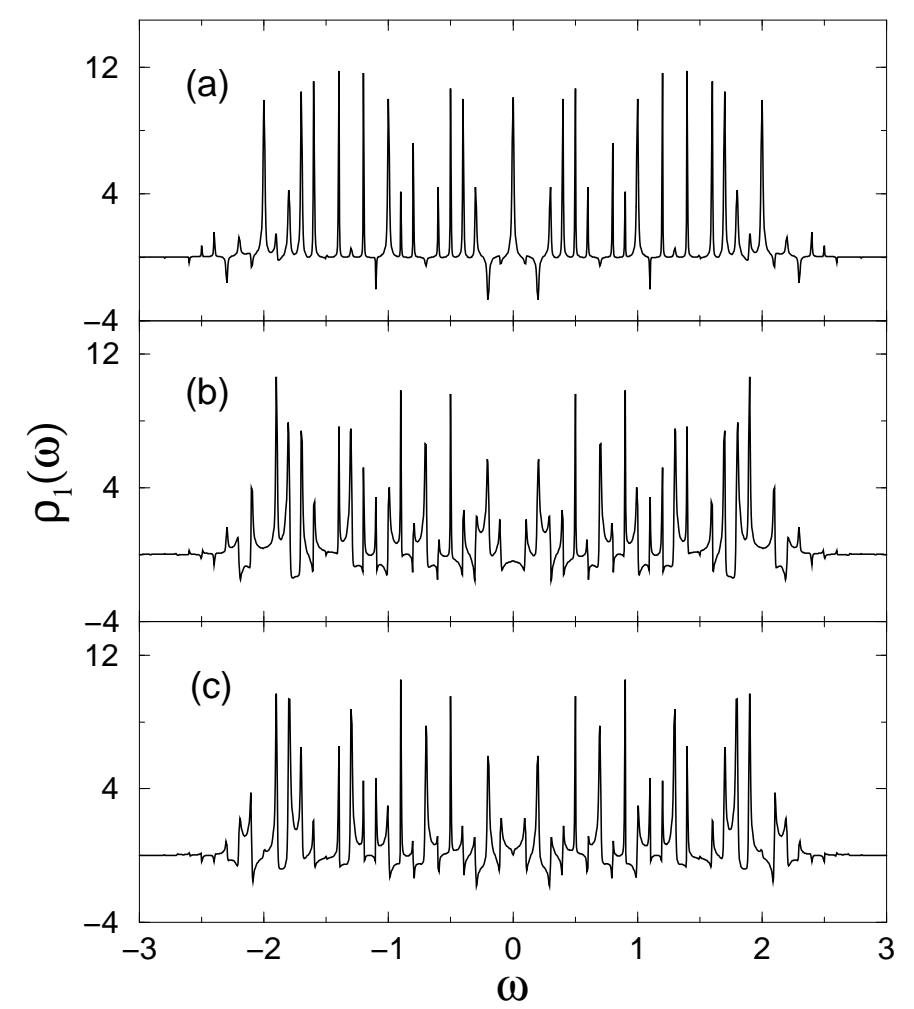

FIG. 7. The generalized time-dependent density of states for a chain of $N=20$ sites with $\hbar \Phi / \Phi_{0}=0.5 W \sigma=0.025 W$ at different times $t=0,1,2$ ( $\mathrm{a}, \mathrm{b}$ and $\mathrm{c}$, respectively).

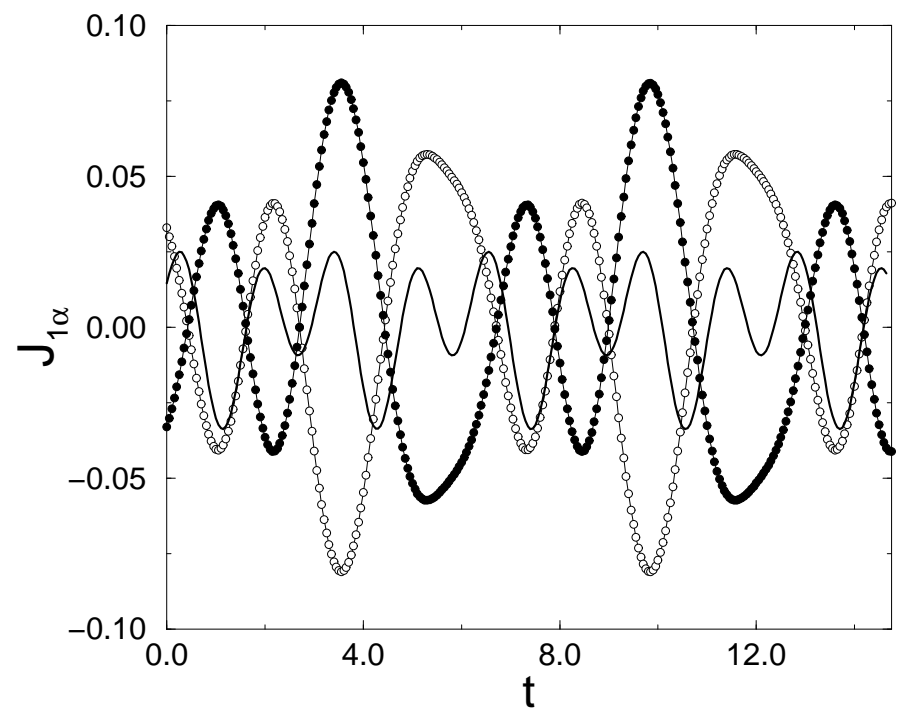

FIG. 8. The current $J_{1 \alpha}(t)$ between the ring and the reservoir as a function of time for a ring with $L=20$ sites and a self-energy $\sigma=0.2 W$. Circles correspond to $\hbar \Phi / \Phi_{0}=0.25 W$ for a chemical potential of the reservoir $\mu=0.25 W$ (solid circles) and $\mu=-0.25 W$ (open circles). The solid line corresponds to $\hbar \Phi / \Phi_{0}=0.5 W$ with $\mu=-0.25 W$. 


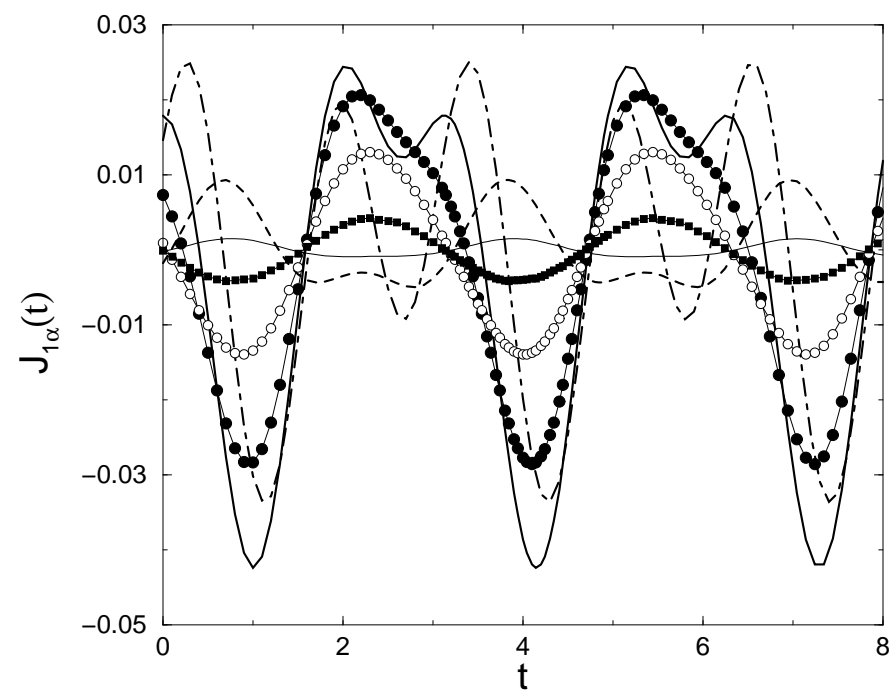

FIG. 9. The current $J_{1 \alpha}(t)$ between the ring and the reservoir as a function of time for a ring with $L=20$ sites. The chemical potential of the reservoir is $\mu=-0.25 \mathrm{~W}$ and $\hbar \Phi / \Phi_{0}=0.5 \mathrm{~W}$. The thin solid, dashed, dot-dashed and thick solid lines, filled circles, opaque circles and filled squares correspond to $\sigma=0.0025 \mathrm{~W}, 0.025 \mathrm{~W}, 0.2 \mathrm{~W}, 0.5 \mathrm{~W}, \mathrm{~W}, 2.5 \mathrm{~W}, 12.5 \mathrm{~W}$, respectively.

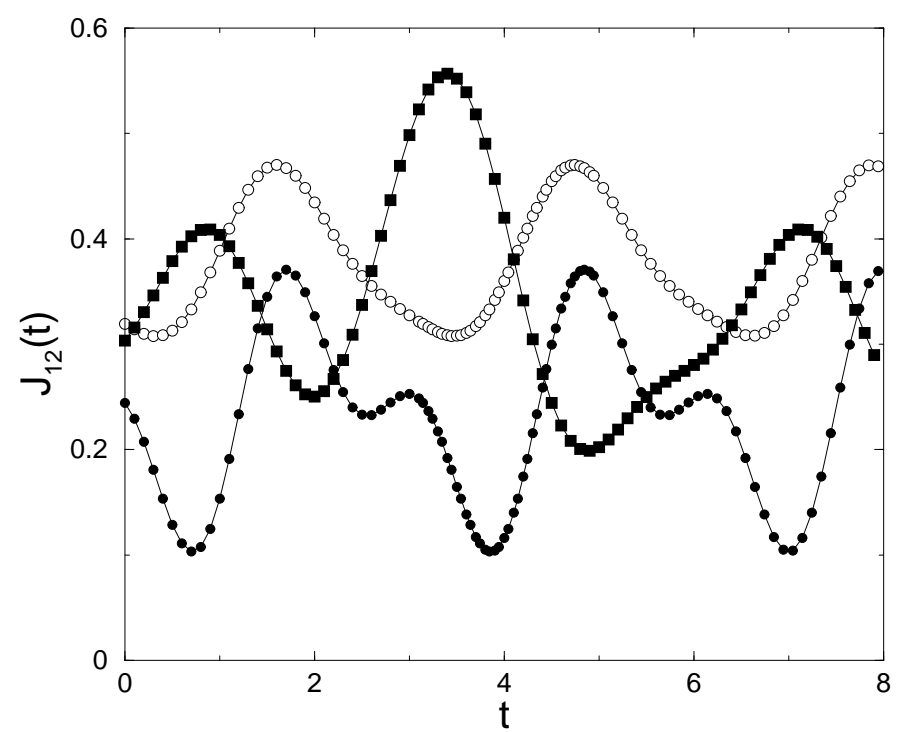

FIG. 10. The current $J_{12}(t)$ along a ring with $L=20$ sites and $\sigma=0.2 W$. Circles correspond to $\hbar \Phi / \Phi_{0}=0.5 W$ and a chemical potential $\mu=0$ and $\mu=-0.25 \mathrm{~W}$ for opaque and filled symbols respectively. Squares correspond to $\hbar \Phi / \Phi_{0}=0.25 W$ and $\mu=-0.25 W$. 


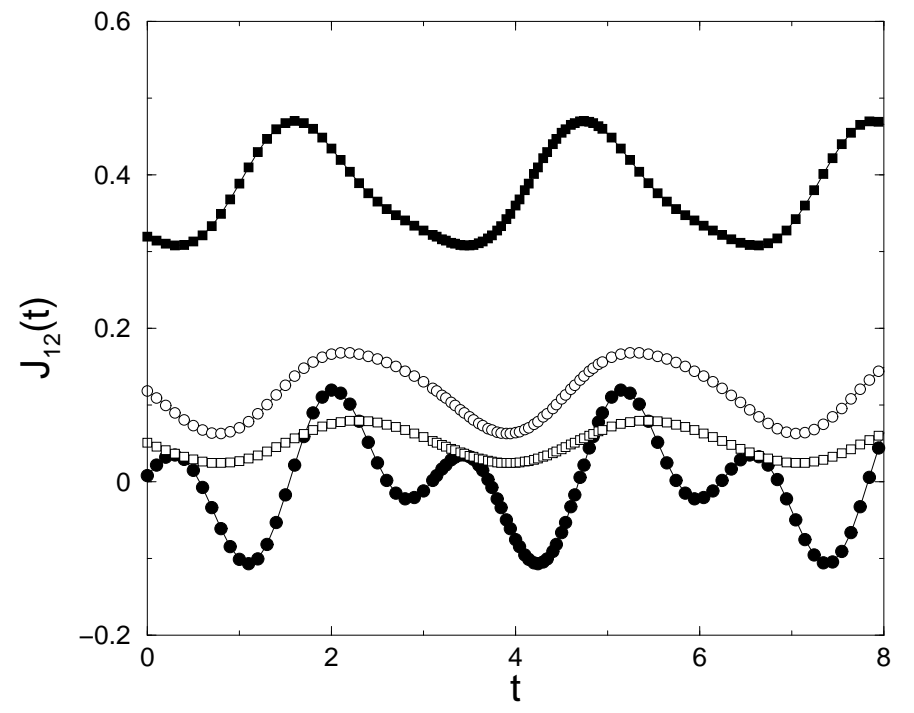

FIG. 11. The current $J_{12}(t)$ along a ring with $L=20$ sites and $\hbar \Phi / \Phi_{0}=0.5 W$ and $\mu=-0.25 W$. Filled circles, filled squares, open circles and open squares correspond to $\sigma=0.0025 W, 0.2 W, W, 2.5 W$, respectively.

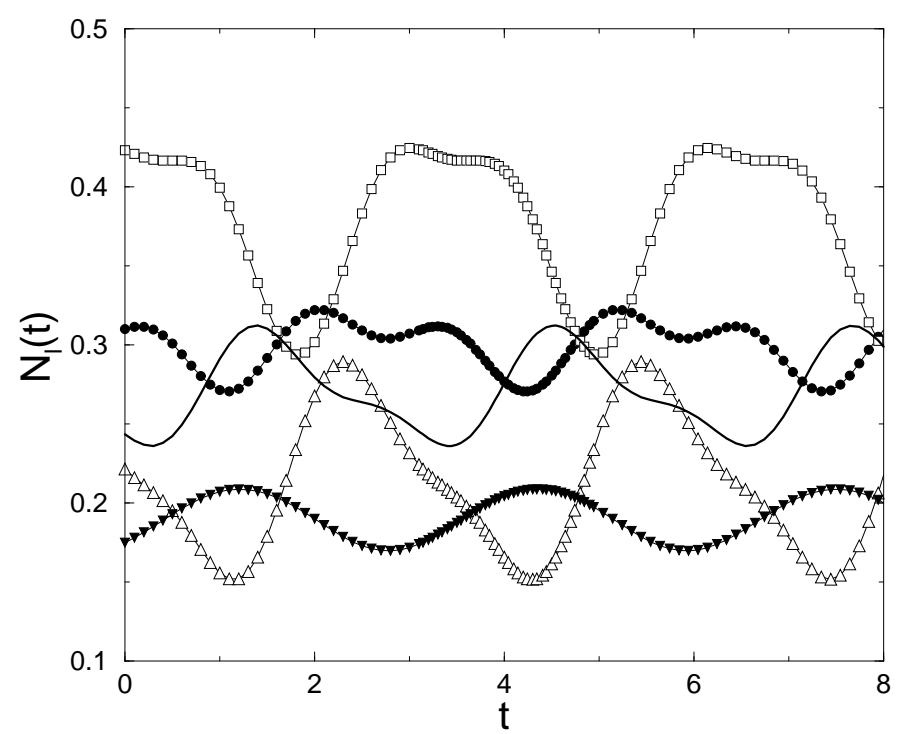

FIG. 12. The occupation number $N_{l}(t)$ along a ring with $L=20$ sites and $\hbar \Phi / \Phi_{0}=0.5 W$ and $\mu=-0.25 W$ and $\sigma=0.2 W$. Circles, squares, up triangles, down triangles and solid line correspond to $l=1,4,12,15,20$, respectively. 


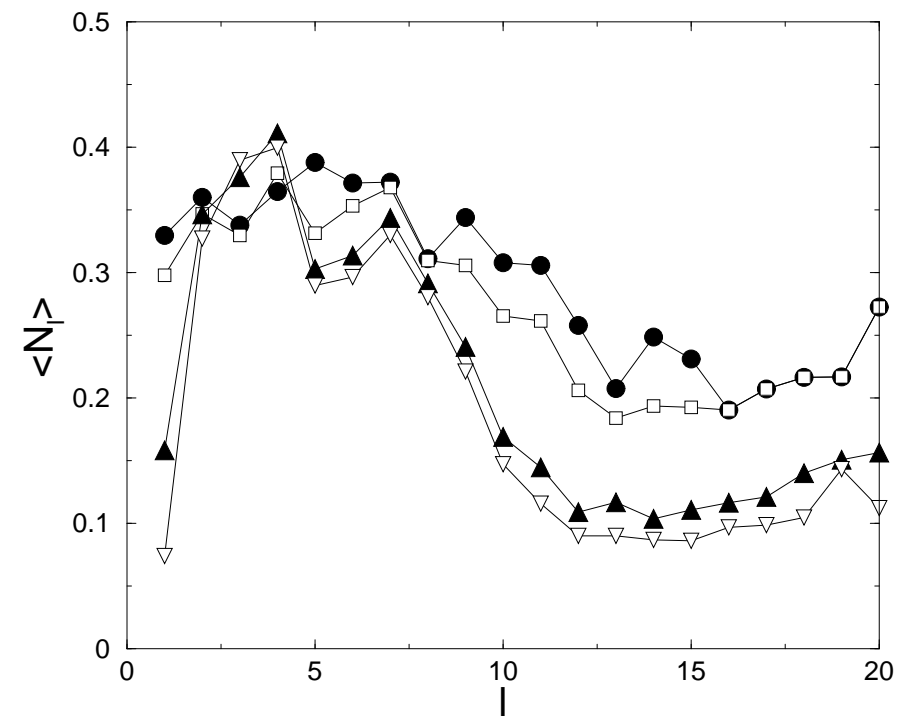

FIG. 13. The mean occupation number $\left\langle N_{l}\right\rangle$ along a ring with $L=20$ sites and $\hbar \Phi / \Phi_{0}=0.5 W$ and $\mu=-0.25 W$. Circles, squares, up triangles, down triangles and solid line correspond to $\sigma=0.0025 W, 0.2 W, W, 2.5 W$, respectively. 
(a)
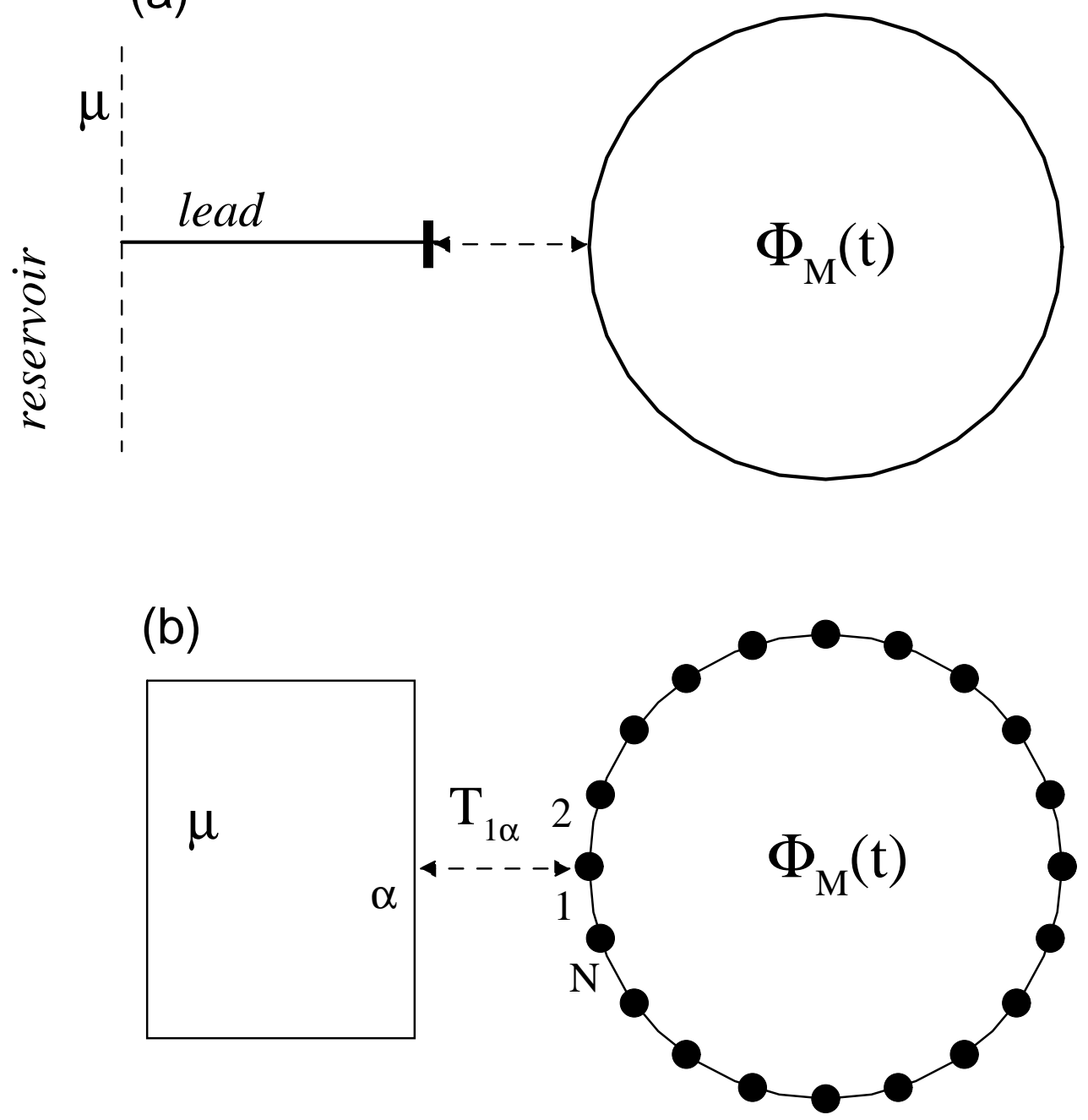